C2006 IEEE. Personal use of this material is permitted. However, permission to reprint/republish this material for advertising or promotional purposes or for creating new collective works for resale or redistribution to servers or lists, or to reuse any copyrighted component of this work in other works must be obtained from the IEEE. 


\section{Quantifying Risk in Financial Terms in an e-Transaction}

\author{
Omar Khadeer Hussain, Elizabeth Chang and \\ Farookh Khadeer Hussain \\ School of Information Systems \\ Curtin University of Technology \\ Perth, AUSTRALIA \\ \{Omar.Hussain, Elizabeth.Chang, \\ Farookh.Hussain\}@cbs.curtin.edu.au
}

\begin{abstract}
An outcome of Risk is the possible loss that could incur in an interaction. In a peer-to-peer financial interaction, the possible loss that could incur is usually the financial loss in the resources of the trusting agent that are involved in the interaction. Hence, a consideration for the trusting agent to analyze the Risk in interacting with any probable trusted agent in order to decide whether to interact with it or not, is to determine the potential loss in its resources that may occur. In this paper, we will propose a methodology by which the trusting agent can determine beforehand the possible loss that could be incurred to it as a result of interacting with a probable trusted agent.
\end{abstract}

\section{INTRODUCTION}

Before proceeding in an interaction, the trusting agent would like to analyze the level of Risk that could be present in interacting with a probable trusted agent. Analyzing the possible level of Risk would considerably help the trusting agent to determine the possible loss in its resources through undertaking the interaction with a probable trusted agent. Further based on that analysis the trusting agent can make an informed decision of whether to interact with that particular trusted agent or not. The Australian and New Zealand Standard on Risk Management, AS/NZS 4360:2004 states that Risk Identification is the heart of Risk Management [1]. Hence, Risk should be identified in order to analyze and manage it better. Risk analysis is the science of evaluating Risks resulting from past, current, anticipated or future activities. The use of these evaluations includes providing information for determining regulatory actions to limit Risk and for decision making. Risk is a combination of:

a) The uncertainty of the outcome; and

b) The cost of the outcome when it occurs.

In the literature, different definitions have been defined for Risk. But all those definitions highlight and emphasize on one factor, the possible loss in an interaction. To mention some such definitions; March et al. define Risk more by the magnitude of the value of the outcome rather than by its likelihood [2]. Luhmann defines Risk in a transaction as where the possible damage might be more than the advantage sought [3]. Mayer et al. conclude that Risk is present in the transaction only if the negative outcome outweighs the positive outcome [4]. In contrast to this definition Rousseau et al. measure Risk as the potential negative consequence and probability of failure [5]. Sztompka defines Risk as the probability of the loss of the resources invested [6]. Grazoli et al. view Risk as the consumers' perception of uncertainty

\author{
Tharam S. Dillon \\ Faculty of Information Technology \\ University of Technology, Sydney \\ Sydney, AUSTRALIA \\ tharam@it.uts.edu.au
}

and adverse consequences of engaging in an activity [7]. Cheung et al. define Risk as having two dimensions; one related to the uncertainty or probability of loss notion and the other related to a consequence of the importance of the notion of loss [8]. We define Risk in the context of peer-topeer business interaction as the likelihood that the trusted agent might not act as expected according to the trusting agent's expectations in a given context and at a particular time once the interaction begins, resulting in financial loss in the resources involved in the interaction [9].

Hence, one of the main decision making factors for the trusting agent to decide whether to interact with a probable trusted agent or not is by determining the possible loss in its resources through the interaction. In a peer-to-peer financial interaction, the possible loss in the resources relates to the financial loss that could be incurred by the trusting agent in interacting with a trusted agent. In this paper, we propose a methodology by which the trusting agent analyzes the level of Risk beforehand by determining the possible loss to its resources in interacting with a trusted agent. This paper is organized in 6 sections. In section 2 we discuss about our previous work of analyzing Risk in an interaction. In section 3 and section 4 we define the factors and propose the methodology for determining the possible financial loss in an interaction. In section 5 we explain the methodology by taking a real world example and finally in section 6 we conclude the paper.

\section{RELATED WORK}

In order to analyze the level of Risk that could be present in an interaction we defined the term Riskiness in Hussain et al [9]. Riskiness is defined as the numerical value that is assigned to the trusted agent on the Riskiness scale by the trusting agent after its interaction. This numerical value shows the level of Risk that was present in their interaction. The Riskiness scale, as shown in figure 1, depicts different levels of Risk that could be possible in an interaction. The Riskiness value is assigned to the trusted agent by the trusting agent after assessing the level of un-commitment in its actual behaviour with respect to the expected behaviour. Expected behaviour is the commitment that the trusted agent was supposed to show in the interaction. The actual behaviour is the actual commitment by the trusted agent in its interaction with the trusting agent. The methodology by which the trusting agent determines the un-committed behaviour by the trusted agent in the interaction and assigns it with a Riskiness value that it deserves is defined in Hussain et al [9]. 


\begin{tabular}{|c|c|c|c|}
\hline $\begin{array}{l}\text { Riskiness } \\
\text { Levels }\end{array}$ & $\begin{array}{c}\text { Magnitude of } \\
\text { Risk }\end{array}$ & $\begin{array}{l}\text { Riskiness } \\
\text { Value }\end{array}$ & Star Rating \\
\hline Unknown Risk & . & -1 & Not Displayed \\
\hline Totally Risky & $\begin{array}{c}91-100 \% \text { of } \\
\text { Risk }\end{array}$ & 0 & Not Displayed \\
\hline $\begin{array}{l}\text { Extremely } \\
\text { Risky }\end{array}$ & $\begin{array}{l}71-90 \% \text { of } \\
\text { Risk }\end{array}$ & 1 & From $2 \mathbb{t}$ to \\
\hline Largely Risky & $\begin{array}{l}51-70 \% \text { of } \\
\text { Risk }\end{array}$ & 2 & From $\lesssim \mathcal{W}$ \\
\hline Risky & $\begin{array}{l}26-50 \% \text { of } \\
\text { Risk }\end{array}$ & 3 & \\
\hline $\begin{array}{l}\text { Largely } \\
\text { UnRisky }\end{array}$ & $\begin{array}{l}11-25 \% \text { of } \\
\text { Risk }\end{array}$ & 4 & From \\
\hline UnRisky & $\begin{array}{c}0-10 \% \text { of } \\
\text { Risk }\end{array}$ & 5 & \\
\hline
\end{tabular}

Figure 1. Showing the Riskiness scale and its associated levels

If the future trusting agent has any previous interaction history with a probable trusted agent in the same time space and in the same context as its future interaction, then it can make a decision whether to interact or not with that particular trusted agent based on its previous interaction history with it, by analyzing the Riskiness value that it assigned to the trusted agent in the past interactions. If, on the other hand, the future trusting agent does not have any previous interaction history with a probable trusted agent, then it can decide whether to interact or not with that particular trusted agent by determining its Riskiness value by soliciting recommendations from other agents in the context of its future interaction. The agents replying back with the recommendations are called Recommending Agents. As mentioned in Hussain et al. [10] the recommending agents communicate their recommendations in the form of a Risk Set. The Risk set is an ordered way of communicating recommendations so that it is easier for the trusting agent to interpret and analyze them. The format of the Risk set is defined in Hussain et al. [10]. Once the future trusting agent receives the recommendations, then it can assimilate them and determine the Riskiness value of the probable trusted agent in question. Based on the Riskiness value achieved, the trusting agent can decide whether to interact or not with it.

However, as mentioned earlier the Riskiness value to a trusted agent is assigned by the trusting agent after assessing the level of un-commitment in its actual behaviour with respect to the expected behaviour. Expected behaviour is defined by the trusting agent according to the criteria of its interaction. Subsequently the Riskiness value that this trusting agent (or recommending agent while communicating recommendations) recommends for the particular trusted agent when solicited for recommendations from other agents is according to its criteria in the interaction. It is highly possible that the future trusting agent analyzing the Riskiness of the particular trusted agent by soliciting recommendations might have different criteria in its interaction as compared to that of the recommending agents even thought it might be in the same context. Recommendations like these will not be of any use to the future trusting agent analyzing the Riskiness of a particular probable trusted agent as they do not match with its criteria even though it is in the same context. Hence, it would be baseless for the future trusting agent to assimilate and determine the Riskiness value of the probable trusted agent from recommendations whose criteria are not similar to those in its interaction.

In order to overcome this disadvantage, we defined a Riskbased Decision Support System (RDSS) in Hussain et al. [11]. Utilizing this system, the trusting agent classifies the recommendations according to its trustworthiness and then assimilates the trustworthy and unknown recommendations according to their time and the criteria in its future interaction with the possible trusted agent. The Riskiness value that the future trusting agent receives for a probable trusted agent is according to the criteria of its future interaction with it. Further, it omits the untrustworthy recommendations and reduces the Risk of assimilating the data which is not trustworthy.

If the future trusting agent has to decide on an agent to interact with among a set of probable trusted agents, then it can use the RDSS to determine the Riskiness value of each agent according to the criteria of its future interaction and then decide on one among them. But it is possible that the Riskiness value of each probable trusted agent determined by RDSS might be the same for one or more trusted agents. Then the basis for the future trusting agent to decide with which agent to interact with, shifts to another characteristic of Risk namely the possible loss that could be incurred. As mentioned before in a peer-to-peer financial interaction, the possible loss to the trusting agent is the financial loss in its resources in interacting with the trusted agent. The trusting agent can analyze the Risk and decide on with which agent to interact with by determining the possible loss to its resources in interacting with each probable trusted agent.

The financial loss to a trusting agent in an interaction is dependent on:

1. Its previous interaction history with the trusted agent, if any, or on the reputation of the trusted agent determined by the trusting agent by soliciting recommendations from other agents according to the criteria of its interaction;

2. The future Riskiness value of the trusted agent predicted by the trusting agent in the time space of it's future interaction with it;

3. Willingness of the trusting agent in interacting with the trusted agent;

4. Familiarity of the trusting agent with the medium of interaction;

5. Familiarity of the trusting agent with the trusted agent; and

6. Nature of the trusting agent.

All these factors have to be considered when determining the possible financial loss in the trusting agent's resources in interacting with the trusted agent. In the next section, we will explain each of these factors in detail and define the metrics which are used to quantify each of these factors and express them numerically.

\section{FACTORS FOR DETERMINING FINANCIAL LOSS IN AN INTERACTION}

\section{A. Previous interactions or considering reputation from other agents}

The outcome of previous interactions, if any, between the future trusting agent and the probable trusted agent will help 
in analyzing the level of Risk that could be present in future interactions between them. Depending on the outcome of the previous interaction, the trusting agent might be able to make a decision to trust the trusted agent or not. If the outcome of the previous interaction was positive and it concluded according to expected behaviour, then the trusting agent might have some belief in the trusted agent and may proceed with any future interactions. Consequently, the Risk associated with this interaction might not be high, as there is some belief present among them. On the contrary, if the outcome of the previous interaction was negative then the trusting agent may have some doubts about proceeding with any future interaction with the trusted agent and fears the Risk involved in the interaction may be too high.

If there is no previous interaction history between the trusting and the probable trusted agents, then the trusting agent can analyze the Risk that could be present in its interaction with each agent by enquiring about its reputation in the particular context of its future interaction. As discussed earlier, reputation of a trusted agent can be determined by soliciting for recommendations from other agents and later assimilating the information. The methodology by which the trusting agent assimilates the recommendations according to its criteria is defined in Hussain et al [11]

We represent the Riskiness value of the probable trusted agent before starting an interaction, that is achieved either by the previous interaction history of the trusting agent with the trusted agent or by the trusting agent soliciting for its recommendations from other agents by the metric Pre

The value of the metric Pre ${ }_{\text {Trusted Agent }}$ is between $(0,5)$ on the Riskiness scale.

\section{B. Predicting the future Riskiness value of the trusted agent}

Risk varies according to time. It is not possible for a trusting agent to have the same impression of the trusted agent that it had at a particular time throughout all interactions. The trusting agent, in order to determine the possible financial loss in an interaction accurately should take into consideration the future Riskiness value of the probable trusted agent till the time space of their interaction. The future Riskiness value for the probable trusted agent is predicted based on either the recommendations acquired from the recommending agents according to the criteria of the trusting agent's future interaction or the previous interaction of the trusting agent with the probable trusted agent in the same context and time space as its future interaction. The process of classifying the time of the trusting agent's interaction with the probable trusted agent into different timeslots and predicting the future Riskiness value of the trusted agent in each timeslot has been defined in Hussain et al. [12].

We represent the future Riskiness value of the probable trusted agent within the given space of time of the trusting agent's interaction by the metric Fut ${ }_{\text {Tusted Agent. }}$ The predicted future Riskiness value of the probable trusted agent Fut ${ }_{\text {Trusted }}$ Agent will be in the range of $(0,5)$ on the Riskiness scale.

C. Willingness of the trusting agent to interact with the trusted agent
The financial loss in an interaction also depends on the commitment / willingness of the trusting agent in dealing with the probable trusted agent. While choosing an agent to interact with from a set of probable trusted agents, it is possible that the trusting agent might have a favourable past interaction history with a particular trusted agent, in the context of its future interaction and as a result of that, it might prefer to choose and deal with that particular trusted agent in its future interaction among the other probable agents. Hence the trusting agent while determining the financial loss in its resources in interacting with a probable trusted agent should consider its willingness in interacting with that probable trusted agent.

We define the willingness of the trusting agent to interact with the probable trusted agent by the metric Will ${ }_{\text {Interaction }}$ In order to measure the willingness of the trusting agent in interacting with the trusted agent we define 3 levels of the metric Will Tusting Agent. Those levels are defined in Table 1.

TABLE 1

SHOWING THE LEVELS FOR THE METRIC WILL INTERACTION $_{\text {N }}$

\begin{tabular}{|c|l|}
\hline $\begin{array}{c}\text { Metric Will Trusting Agent } \\
\text { Levels }\end{array}$ & \multicolumn{1}{c|}{ Semantics of the level } \\
\hline 0 & $\begin{array}{l}\text { The trusting agent is not at all willing to deal } \\
\text { with the trusted agent }\end{array}$ \\
\hline 1 & $\begin{array}{l}\text { The trusting agent is comfortable in dealing } \\
\text { with the trusted agent but would prefer in } \\
\text { dealing with other agents if possible }\end{array}$ \\
\hline 2 & $\begin{array}{l}\text { The trusting agent is totally comfortable in } \\
\text { dealing with the trusted agent }\end{array}$ \\
\hline
\end{tabular}

D. Familiarity of the trusting agent with the medium of interaction

The possible degree of financial loss in the trusting agent's resources also varies according to the familiarity of the trusting agent towards the medium of interaction with the probable trusted agent. If the trusting agent is familiar with the medium in which it is going to interact then it will ease the Risk that could be present.

We represent the familiarity of the trusting agent with the medium of interaction by the metric Fam medium. In order to measure the familiarity of the trusting agent with the medium of interaction we define two levels for the metric Fam ${ }_{\text {Medium }}$ Those levels are defined in Table 2.

TABLE 2

SHOWING THE LEVELS FOR THE METRIC FAM

\begin{tabular}{|c|l|}
\hline $\begin{array}{c}\text { Metric Fam } \\
\text { Levels }\end{array}$ & \multicolumn{1}{|c|}{ Semantics of the level } \\
\hline 0 & $\begin{array}{l}\text { The trusting agent is not familiar with the } \\
\text { medium of the interaction }\end{array}$ \\
\hline 1 & $\begin{array}{l}\text { The trusting agent has previous experience and is } \\
\text { familiar with the medium of the interaction }\end{array}$ \\
\hline
\end{tabular}

\section{E. Familiarity of the trusting agent with the trusted agent}

In order to determine the potential financial loss in the trusting agent's resources in an interaction it is important to consider the familiarity of the trusting agent with the particular trusted agent. The familiarity of the trusting agent with the trusted agent is represented by the metric Fam ${ }_{\text {Trusted }}$ 
Agent In order to measure the familiarity of the trusting agent with the trusted agent we define two levels of the metric Fam Trusted Agent as shown in Table 3.

TABLE 3

SHOWING THE LEVELS FOR THE METRIC FAM TRUSTED AGENT $_{\text {TE }}$

\begin{tabular}{|c|l|}
\hline $\begin{array}{c}\text { Metric } \\
\text { Fam } \\
\text { Levested Agent }\end{array}$ & \multicolumn{1}{c|}{ Semantics of the level } \\
\hline 0 & $\begin{array}{l}\text { The trusting agent has not interacted with the } \\
\text { trusted agent before and is not at all familiar with it }\end{array}$ \\
\hline 1 & $\begin{array}{l}\text { The trusting agent has previous interaction history } \\
\text { with the trusted agent and is familiar with it. }\end{array}$ \\
\hline
\end{tabular}

\section{$F$. Nature of the trusting agent}

One of the factors which vary Risk in an interaction is the nature and thinking attitude of the trusting agent, which decides on how it will act and react in certain situations. The nature of the trusting agent plays an important role in deciding whether to proceed with the interaction or not, thus helping in determining the financial loss that could be involved in an interaction.

If the trusting agent has an impatient nature or acts in haste, then there is a possibility that he might proceed with the interaction without looking at the previous history of the probable trusted agent or soliciting for recommendations, hoping to achieve the desired outcome as soon as possible. The Risk associated in these interactions might be high.

The trusting agent might care for personal values and start an interaction. For example let us suppose that the trusting agent and the trusted agent might have dealt successfully before in a different context and in a different interaction. Now, they are dealing again in a context and in an interaction in which the trusted agent does not have any experience. Yet the trusting agent might care for personal values and might be ready to take the extra Risk involved and proceed with the interaction.

On the contrary, the trusting agent might have a cautious nature of proceeding according to the results and feedback of the previous interactions of the trusted agent. In this case, it might not be willing to trust the trusted agent in any other context in which it does not have any experience. He might feel the Risk involved in such an interaction could be high and might not proceed in interacting with him. Hence, the Risk too decreases according to the trusting agent's decision. If the trusting agent decides to go ahead in an interaction according to the correct way, i.e. in a logical fashion not caring about the personal values then it will be making the decision to proceed with the interaction only if the expected advantages outweigh the negative factors. The Risk involved in such interactions might be less when compared to the other ones.

The trusting agent might decide to proceed in the interaction or not, depending on its nature and it is important to take that into consideration while determining the financial loss in an interaction. The nature of the trusting agent is represented by the metric Nat Trusting Agent . We define 3 levels of the metric Nat ${ }_{\text {Trusting Agent }}$ in order to determine the nature of the trusting agent. Those levels are defined in Table 4.
TABLE 4

SHOWING THE LEVELS FOR THE METRIC NAT

\begin{tabular}{|c|l|}
\hline $\begin{array}{c}\text { Metric } \\
\text { Nat } \\
\text { Trusting Agent } \\
\text { Levels }\end{array}$ & \multicolumn{1}{c|}{ Semantics of the Level } \\
\hline 0 & $\begin{array}{l}\text { The trusting agent is impulsive in nature and acts in } \\
\text { haste. It prefers proceeding in an interaction even if } \\
\text { there is high Risk or without analyzing the Risk that } \\
\text { could be present in an interaction. }\end{array}$ \\
\hline 1 & $\begin{array}{l}\text { The trusting agent is conservative in nature and } \\
\text { prefers taking some Risk. It might also care for } \\
\text { personal values. }\end{array}$ \\
\hline 2 & $\begin{array}{l}\text { The trusting agent is determined in nature and will } \\
\text { proceed in the interaction only if the expected } \\
\text { advantages outweigh the negative factors. }\end{array}$ \\
\hline
\end{tabular}

\section{DETERMINING THE POSSIBLE FINANCIAL LOSS} IN AN INTERACTION

To determine the possible financial loss in an interaction, the trusting agent by making use of the above mentioned metrics should derive a numerical value which quantifies its possible interaction with a particular trusted agent. The numerical value that is derived by using the above metrics will take into consideration factors namely

- the previous interaction of the trusting agent with the trusted agent if any,

- the reputation of the trusted agent determined by assimilating the recommendations,

- the predicted future Riskiness value of the trusted agent within the time slot of its interaction with the trusting agent,

- the willingness of the trusting agent in dealing with the trusted agent,

- familiarity of the trusting agent with the medium of interaction,

- familiarity of the trusting agent with the trusted agent,

- nature of the trusting agent which is a critical factor in deciding whether to interact or not with the trusted agent.

Hence the numerical value quantifying the possible interaction of the trusting agent with a probable trusted agent can be determined by adding the individual value that is assigned for each metric. We represent the numerical value quantifying the possible interaction of the trusting agent with the trusted agent as Poss

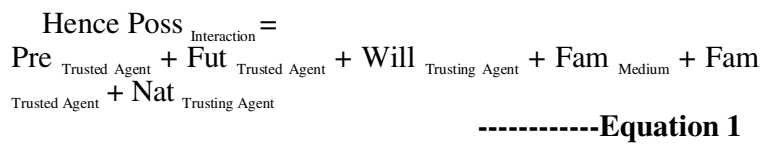

Once the numerical value quantifying the possible interaction between the trusting agent and the trusted agent has been determined, it should be compared with the numerical value which quantifies an interaction between the same agents with no financial loss at all to the trusting agent's resources. This value is achieved by the above defined metrics by substituting the values for each metric in equation 1 that would represent a totally non-risky interaction. We represent the numerical value which quantifies an interaction with no financial loss at all to the trusting agent's resources as Noloss 


$$
\begin{aligned}
& \text { Hence Noloss } \\
& \text { Pre }_{\text {Trusteraction Agent }}+\text { Fut }_{\text {Trusted Agent }}+\text { Will } \\
& \text { Trusting Agent } \\
& \text { Trusted Agent }_{\text {Trusting Agent }}+\mathrm{Fam}_{\text {Medium }}+\text { Fam } \\
& \text {------------Equation } 2
\end{aligned}
$$

We define Loss as the metric which expresses in numerical value the possible level of loss that could be possible in an interaction. This is achieved by comparing the difference of the numerical values representing a totally nonrisky interaction and the possible way in which the interaction might proceed with respect to the numerical value that expresses an interaction with no financial loss at all. The metric Loss ${ }_{\text {Interaction }}$ depicts the level of financial loss that could be possible in an interaction to the trusting agent with the trusted agent.

$$
\begin{gathered}
\text { Hence Loss } \\
\text { Loss }_{\text {Interaction }} \text { is deterion } \\
=\frac{\text { NolossInteraction }- \text { Possinteraction }}{\text { Nolossinteraction }}
\end{gathered}
$$

Equation 3

Consequently, Financial Loss in an interaction $=$

(Cost of the Interaction * Loss

Equation 4

The possible percent of loss in an interaction $=$

$$
\begin{aligned}
& \text { Possible Percent of Loss } \\
& \text { Interaction }=\left(\operatorname{Loss}_{\text {Interaction }} * 100\right) \\
&--------------- \text { Equation } 5
\end{aligned}
$$

\section{EXAMPLE OF DETERMINING THE POSSIBLE FINANCIAL LOSS IN AN INTERACTION}

In order to get a better understanding of the proposed model let us consider an example of determining the possible financial loss in an interaction to the trusting agent in interacting with a logistic company which would be the trusted agent by using the defined metrics.

Let us consider that a trusting agent ' $\mathrm{A}$ ' has to interact with a logistic company in the context of 'transporting its goods'. The trusting agent has to decide among a set of logistic companies (possible trusted agents) with which particular agent to interact with. The set of possible trusted agents are Agent ' $\mathrm{B}$ ' and Agent ' $\mathrm{C}$ '. Let us further assume that

- The criteria in the trusting agent's future interaction are $\mathrm{C} 1, \mathrm{C} 2$ and $\mathrm{C} 3$,

- The goods are of worth $\$ 15,000$,

- The trusting agent wants to interact with the trusted agent in the period of 01/02/2006 to 05/02/2006, and

- The trusting agent ' $A$ ' had interacted favourable with the trusted agent ' $\mathrm{B}$ ' previously in the same context but at a different time slot.

In order for the trusting agent to decide with which agent to interact with, it will divide the time space into different time slots and determine the Riskiness value or the reputation of each possible trusted agent in the time slots according to its criteria by utilizing the Risk based Decision Support System (RDSS) till the time spot of its interaction. The process of the trusting agent dividing the time into different time slots and determining the Riskiness values or the reputation of the possible trusted agents according to the criteria of its future interaction by either considering its previous interactions with the trusting agent or by assimilating recommendations from the recommending agents is mentioned in Hussain et al [11]. That Riskiness value is represented by the metric Pre

For explanation sake let us consider that:

- The value of the metric Pre ${ }_{\text {Tusted Agent }}$ determined by the trusting agent for the possible trusted agents ' $\mathrm{B}$ ' and ' $\mathrm{C}$ ' is 4 and 4 respectively.

Based on the Riskiness values determined for the possible trusted agents in each previous time slots by RDSS, the trusting agent can then predict the future Riskiness value or the reputation of those agents within the time space of its interaction by utilizing the methodology mentioned in Hussain et al [12]. The future Riskiness value is represented by Fut ${ }_{\text {Tusted Agent }}$

Again for explanation sake let us consider that:

- The future Riskiness values predicted by the trusting agent from the previous time slots Riskiness values for the trusted agents ' $\mathrm{B}$ ' and ' $\mathrm{C}$ ' is 4 and 4 respectively.

Hence as the past and the future Riskiness values of both the possible trusted agents in the time space of the trusting agent's future interaction is the same, it will be difficult for the trusting agent to decide with which particular agent to interact with. It can ease its decision making process through analysing the possible Risk present by considering the degree of possible financial loss to it in interacting with each of them.

G. Determining the Financial loss to the trusting agent in interacting with possible trusted agent ' $B$ '

Utilizing the above defined metrics to determine the financial loss to the trusting agent ' $\mathrm{A}$ ' in interacting with the trusted agent ' $\mathrm{B}$ ':

Pre ${ }_{\text {Trusted Agent } \mathrm{B} \text { ' }}=4$

Fut $_{\text {Trusted Agent }}{ }^{B} \cdot=4$

Since the trusting agent ' $A$ ' has a favourable interaction history with trusted agent ' $\mathrm{B}$ ' it assigns a value of 2 to the metric Will Tusting Agent $_{\text {a }}$

As the trusting agent ' $\mathrm{A}$ ' has previous interaction history with the trusted agent ' $\mathrm{B}$ ' it is familiar with the medium of interaction. Hence the value of Fam Medium $_{\text {is }} 1$

The value of Fam Trusted Agent $_{\text {is } 1}$ ing

The trusting agent is determined in nature and the value for the metric Nat ${ }_{\text {Tusting Agent }}$ is 2 .

Quantifying the possible interaction of the trusting agent 'A' with the trusted agent ' $\mathrm{B}$ ' according to equation 1 we get: Poss ${ }_{\text {Interaction ' } \mathrm{B} \text { ' }}=14$

Quantifying the value of the metric Noloss ${ }_{\text {Interaction }}$ that would represent a non-Risky interaction hence no loss in financial terms to the trusting agent by using equation 2 we get: 


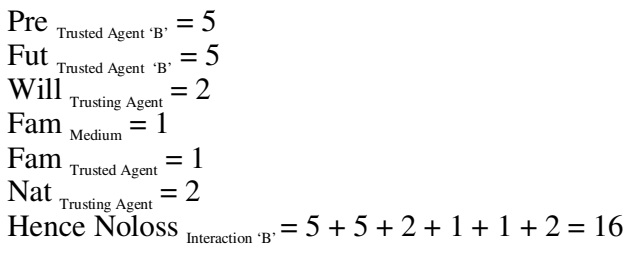

Determining the numerical value expressing the possible degree of loss that could be possible in an interaction by utilizing equation 3 we get:

Loss $_{\text {Interaction } \mathrm{B}^{\prime}}=\frac{16-14}{16}=\mathbf{0 . 1 2 5}$

Consequently determining the Financial Loss in an interaction according to equation 4

Financial Loss in interacting with agent ' $B$ ' $(15000 * 0.125)=\$ 1875$ Possible Percent of Loss ${ }_{\text {Interaction 'B' }}=\left(\operatorname{Loss}_{\text {Interaction ' }^{B} \text { ' }} * 100\right)$
Possible Percent of Loss ${ }_{\text {Interaction }{ }^{\mathrm{B}} \text { ' }}=12.5 \%$

H. Determining the Financial loss to the trusting agent in interacting with possible trusted agent ' $C$ '

Similarly determining the possible financial loss to the trusting agent ' $A$ ' by utilizing the metrics in interacting with the trusted agent ' $\mathrm{C}$ ' we get:

Pre ${ }_{\text {Trusted Agent } \mathrm{C}^{\prime}}=4$

Fut ${ }_{\text {Trusted Agent } \text { C }^{\prime}}=4$

Although the trusting agent ' $A$ ' has not interacted previously with the trusted agent ' $\mathrm{C}$ ', it is comfortable in dealing with it by analyzing its past and future Riskiness values. But it would prefer in dealing with agent ' $\mathrm{B}$ ' as it has a previous interaction history with it. Hence it assigns a value of 1 to the metric Will

The trusting agent ' $\mathrm{A}$ ' has previous interaction history in this type of medium and hence the value of Fam ${ }_{\text {Medium }}$ is 1

As the trusting agent ' $A$ ' does not have any previous interaction history with the trusting agent ' $C$ ' the value of the metric Fam Trusted Agent $_{\text {is } 0}$

The trusting agent is determined in nature and the value for the metric Nat ${ }_{\text {Trusting Agent }}$ is 2 .

Quantifying the possible interaction of the trusting agent with the trusted agent by using equation 1 we get:

Poss ${ }_{\text {Interaction }{ }^{~} \mathrm{C}}=12$

Quantifying the value of the metric Noloss ${ }_{\text {Interaction }}$ that would represent a non-Risky interaction and hence no loss in financial terms to the trusting agent by using equation 2 we get:

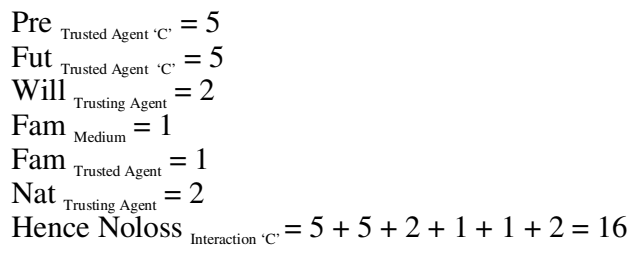

Utilizing equation 3 to determine in numerical terms the degree of possible loss that could be possible in the interaction we get:

Loss $_{\text {Interaction }{ }^{C} \text { ' }}=\frac{16-12}{16}=\mathbf{0 . 2 5}$

Consequently determining the Financial Loss in an interaction according to equation 4

Financial Loss in interacting with agent ' $C$ '

$$
(15000 * 0.25)=\$ \mathbf{3 7 5 0}
$$

Possible Percent of Loss ${ }_{\text {Interaction }^{C} C}=\left(\right.$ Loss $\left._{\text {Interaction ' } C \text { ' }} * 100\right)$ Possible Percent of Loss Interaction $^{C}$ ' $=25 \%$

Hence the possible loss to the trusting agent ' $A$ ' in interacting with the trusted agents ' $\mathrm{B}$ ' and ' $\mathrm{C}$ ' is 1875 \$ and $3750 \$$ respectively. Based on the values achieved the trusting agent can easily conclude on a decision with which agent to interact with.

\section{CONCLUSION}

In this paper we highlighted an important characteristic of Risk in any interaction, its impact in financial terms on the trusting agent's resources. Once the trusting agent gets an idea of the possible financial loss in its resources in the interaction then it can firm its decision of interacting with the trusted agent or not. In this paper we identified the factors by which the loss in the trusting agent's resources involved in the interaction can be determined. Further we defined the metrics by which those factors can be quantified to determine the financial loss in an interaction. We then defined a methodology by which the trusting agent can determine the possible financial loss in interacting with the trusted agent and finally concluded the paper by explaining the methodology with an example.

\section{REFERENCES}

[1] D.F. Cooper, 'The Australian and New Zealand Standard on Risk Management, AS/NZS 4360:2004', Tutorial Notes: Broadleaf Capital International Pty Ltd. Available: http://www.broadleaf.com.au/tutorials/Tut Standard.

J. G. March and Z. Shapira, 'Managerial perspective on risk and risk taking', Management Science, vol. 33, no. 11, pp. 1404-1418

N. Luhmann, 'Familiarity, confidence, trust: Problems and alternatives', Making and Breaking Cooperative Relations, Basil Blackwell, New York, USA.

R.C. Mayer, J.H. Davis and F.D. Schoorman, 'An interactive model for organizational trust', Academy of Management Review, vol. 20, no. 3, pp.709-734.

D.M. Rousseau, S.B. Sitkin, R.S. Burt and C. Camerer, 'Not so different after all: A crossdiscipline view of trust', Academy of Management Review, vol. 23, no. 3, pp. 391-404. P. Sztompka, 'Trust: A sociological theory', Cambridge University Press, Cambridge, U.K. S. Grazioli and A. Wang, 'Looking without seeing: Understanding unsophisticated consumer success and failure to detect Internet deception', Proceedings of the International Conference C. Chemation Systems, New Orleans, USA, Decenber 16-19200, pp 193-204.

odel and measurement instrument', Proceedings of the 2000 Americas Conference on Information Systems, Long Beach, CA, August 10-13 2000, pp 681-689.

[9] O.K. Hussain, E. Chang, F.K.Hussain and T.S. Dillon, "A Methodology for Risk Measurements in e-Transactions", Special issue of International Journal of Computer System, Science and Engineering, CRL Publishing Ltd UK, 2006, pp. 17-31. O.K Hussain, E.Chang, F.K. Hussain, T.S. Dillon and B. Soh, "Modeling the Risk Relationships and Defining the Risk Set" Proceedings of CollECTeR Latam 2005, Chile, 3-5 October 2005, pp 1-9.

[11] O.K Hussain, E.Chang, F.K. Hussain, T.S. Dillon and B. Soh, "Risk Based Decision Suppon System", Proceedings of the 20 International Conference on Advanced Informatio

[12] Networking and Applications, (AINA 2006), Vienna, April 18-20 2006, pp 585-590. of Risk", Proceedings of the 4th ACS/IEEE International Conference on Computer System and Applications (AICCSA-06), Dubai/Sharjah, UAE, March 8-11 2006, pp 500-507. 